\title{
BMJ Open Qualitative investigation of trace-based communication: how are traces conceptualised in healthcare teamwork?
}

\author{
Sayra Cristancho (D) , ${ }^{1}$ Emily Field ${ }^{2}$
}

To cite: Cristancho S,

Field E. Qualitative

investigation of trace-based communication: how are traces conceptualised in healthcare teamwork? BMJ Open 2020;10:e038406. doi:10.1136/ bmjopen-2020-038406

- Prepublication history and additional materials for this paper is available online. To view these files, please visit the journal online (http://dx.doi. org/10.1136/bmjopen-2020038406).

Received 10 March 2020 Revised 05 0ctober 2020 Accepted 17 0ctober 2020

Check for updates

(c) Author(s) (or their employer(s)) 2020. Re-use permitted under CC BY-NC. No commercial re-use. See rights and permissions. Published by BMJ.

'Department of Surgery, Faculty of Education and Centre for Education Research \& Innovation, Western University, London, Ontario, Canada

${ }^{2}$ Centre for Education Research \& Innovation, Western University, London, Ontario, Canada

Correspondence to Dr Sayra Cristancho; sayra.cristancho@schulich. uwo.ca

\section{ABSTRACT}

Objectives This interview-based qualitative study aims to explore how healthcare providers conceptualise tracebased communication and considers its implications for how teams work. In the biological literature, trace-based communication refers to the non-verbal communication that is achieved by leaving 'traces' in the environment and other members sensing them and using them to drive their own behaviour. Trace-based communication is a key component of swam intelligence and has been described as a critical process that enables superorganisms to coordinate work and collectively adapt. This paper brings awareness to its existence in the context of healthcare teamwork.

Design Interview-based study using Constructivist Grounded Theory methodology.

Setting This study was conducted in multiple team contexts at one of Canada's largest acute-care teaching hospitals.

Participants 25 clinicians from across professions and disciplines. Specialties included surgery, anesthesiology, psychiatry, internal medicine, geriatrics, neonatology, paramedics, nursing, intensive care, neurology and emergency medicine.

Intervention Not relevant due to the qualitative nature of the study.

Primary and secondary outcome Not relevant due to the qualitative nature of the study.

Results The dataset was analysed using the sensitising concept of 'traces' from Swarm Intelligence. This study brought to light novel and unique elements of trace-based communication in the context of healthcare teamwork including focused intentionality, successful versus failed traces and the contextually bounded nature of the responses to traces. While participants initially felt ambivalent about the idea of using traces in their daily teamwork, they provided a variety of examples. Through these examples, participants revealed the multifaceted nature of the purposes of trace-based communication, including promoting efficiency, preventing mistakes and saving face.

Conclusions This study demonstrated that clinicians pervasively use trace-based communication despite differences in opinion as to its implications for teamwork and safety. Other disciplines have taken up traces to promote collective adaptation. This should serve as inspiration to at least start exploring this phenomenon in healthcare.

\section{Strengths and limitations of this study}

The use of Swarm Intelligence as a sensitising framework brought awareness to an alternative form of non-verbal communication.

- This paper offers a novel conceptualisation of tracebased communication in healthcare teamwork.

- Analysis of various examples of traces illustrated the key elements of the definition of a trace: focused intentionality, successful versus failed traces and the contextually bounded nature of the responses.

- As the focus was on providing a definition of a trace, this study did not investigate how trace-based communication is used in combination with verbal communication.

- Sampling clinicians from a variety of specialties offered the opportunity to identify patterns in how traces are conceptualised, but prevented exploring which environments may benefit more than others.

\section{INTRODUCTION}

Clear, explicit, verbal communication has been identified as an important marker of effective teamwork. Hence teamwork training in healthcare has tended to prioritise verbal communication over non-verbal communication. ${ }^{1}$ In the healthcare literature, nonverbal communication in teamwork has mostly been referred to as body language in the form of gesture, posture, bodily orientation, facial expression, eye contact or physical distance. ${ }^{23-9}$ While important, research on non-verbal communication in healthcare teamwork has not been as extensive as on verbal communication. For instance, in the context of surgery, some authors have suggested that the lack of in-depth attention to non-verbal communication is related to the tendency of teams to take non-verbal communication for granted. ${ }^{3}$ Given the limited attention placed on non-verbal communication in the teamwork literature, a paucity of research exists that considers whether other forms of non-verbal communication, beyond body language, might be relevant in healthcare teamwork. Research in biology has identified 
a form of non-verbal communication-referred to as trace-based communication - that non-human organisms (such as social insects) use to effectively work together as a collective. ${ }^{10}$ Using the analogy of social insects, this paper explores how healthcare providers conceptualise trace-based communication and considers its implications for how teams work.

Communication literature within the field of healthcare recognises the inextricable relationship between non-verbal and verbal communication..$^{71-13}$ The communication literature highlights the integral role nonverbal communication plays in the patient-physician encounter. Physician gaze, body posture, hand movements, tone of voice, facial expression and even scrub colour impact how patients perceive their clinical care and the quality of physician's communication. ${ }^{14-18}$ The interaction between verbal and non-verbal communication is so important to the patient-physician exchange, that many medical educators argue for explicitly training clinicians in this skillset. ${ }^{19}$ In healthcare teams, particularly surgery, non-verbal communication enables teams to effectively operate during complex or urgent moments in the surgical encounter. Where one positions their body during a code can help indicate leadership roles ${ }^{7}$ or indicate to a trainee that the surgical staff member wishes to take over the procedure. ${ }^{3}$ Additionally, nonverbal communication is useful tool in navigating team hierarchies. For example, a nurse raising their eyebrows or a trainee intentionally moving a piece of surgical equipment further from a surgeon's grasp may act as an important form of 'speaking up' that both saves face and avoids conflict. $^{252021}$

According to broader communication theories, nonverbal communication encompasses more than just body language and has been defined as 'intentional behaviour that is used to symbolically convey an idea' or 'everything that we do except the words that we use in our face to face interactions... even our artefacts, the clothes we wear, the rings and jewellery that we carry with us' ${ }^{22}$ The use of artefacts is the foundation of trace-based communication. In the biological literature, trace-based communication refers to the non-verbal communication that is achieved by leaving 'traces' in the environment and other members sensing them and using them to drive their own behaviour. ${ }^{23} 24$ Trace-based communication is a key component of swam intelligence and has been described as a critical process that enables superorganisms to coordinate work and collectively adapt. ${ }^{23}$ Ants, bees, fish colonies and human societies are all examples of superorganisms in nature. ${ }^{25}$ In the case of ants, they leave pheromones for other ants to smell and know what to do. For humans, leaving your coat on a chair indicates to others that this seat is occupied. While there is room for multiple interpretations (ie, someone forgot their coat), the object was intentionally used to communicate a message to others.

While trace-based communication is gaining traction in other industries, its exploration and use in healthcare teamwork remain largely unacknowledged. Examples of other industries actively using trace-based communication include the military to improve SEAL (SEa, Air, and Land) team training, ${ }^{26}$ the construction industry to increase the efficiency in interior wall building, ${ }^{27}$ and the tech industry to optimise forecasts and decision-making. ${ }^{28}$ These examples share the same philosophy: without directly talking to each other, individuals become aware of traces left in their work environment and use them to carry out their part of the work. In the case of healthcare, the introduction of electronic health records has opened one avenue for the use of traces such as digital 'flags' for asynchronous communication in acute care settings. ${ }^{29}$ Others are beginning to uncover some versatile non-verbal strategies trainees are using to challenge decisions by their consultants. ${ }^{21}$ For instance, in an intubation emergency, effective cues have included obstructing the consultant, touching the consultant's shoulder repeatedly, raising their hand, removing the laryngoscope or placing the face mask near the consultant. The first three cues used body language, while the latter two featured traces (ie, objects) to communicate a message. The grouping of these two different forms of non-verbal communication into the same category suggests either lack of awareness or lack of language to discriminate them. These incidental research findings are drawing attention to the presence of trace-based communication in clinical settings. Yet a purposeful exploration of trace-based communication in healthcare teamwork is lacking, and thus its potential to strengthen teamwork remains largely untapped.

This paper explores how trace-based communication is conceptualised by healthcare providers and how it might be harnessed for better team function. Unless alternative forms of non-verbal communication are more systematically understood, it is difficult to determine how to support its place and relevance in healthcare teamwork.

\section{METHODS}

Purpose and intention drive all forms of human communications. Understanding how and why someone decides to communicate in a certain way and not another is not easily quantified. It requires an approach to research that promotes direct engagement with the participant that allows participants to make sense of their experiences and that systematically analyses words over numbers. ${ }^{30}$ Qualitative methodologies constitute such an approach. Out of the variety of qualitative methodologies, ${ }^{31}$ this paper uses Constructivist Grounded Theory (CGT) to explore how trace-based communication is conceptualised by healthcare providers. CGT is a qualitative methodology used to understand the ways in which individuals and groups socially interact, conceptualise, adjust and establish relationships and patterns of behaviour. ${ }^{32}$

Since the goal is to explore in-depth and make sense of people's experiences, qualitative research data consist of lengthy accounts of those experiences that can be obtained with relatively small samples. This study 
employed a purposeful sampling strategy. ${ }^{33} 34$ Purposeful sampling involves identifying and selecting individuals that are especially knowledgeable about or experienced with a phenomenon of interest. In selecting these individuals, it is important to consider availability and willingness to participate, and the ability to communicate experiences and opinions in an articulate, expressive and reflective manner. Clinicians from across professions and disciplines were invited to participate in a single, $60 \mathrm{~min}$ semistructured interview; 25 individuals consented to be interviewed for this study. Specialties included surgery, anesthesiology, psychiatry, internal medicine, geriatrics, neonatology, paramedics, nursing, intensive care, neurology and emergency medicine. The first author of this paper, who conducted all interviews, is a $\mathrm{PhD}$ scientist appointed to a clinical department. As a non-clinician, she does not hold any clinical or supervisory relationship with the participants. An interview guide was constructed at the outset of the study and refined in an iterative fashion as per the principles of CGT. During the piloting of the interview guide, it became evident that participants struggled with some of the language related to trace-based communication and its origins; therefore, the decision was made to provide a succinct and accessible summary of the key ideas for contextual purposes. At the start of the interview, participants read the one-page explanation of key principles of Swarm Intelligence (see online supplemental appendix 1) and the following description of trace-based communication: 'A key premise in biology states that when organisms act in a common environment they leave traces. Traces are objects, marks or signals that stimulate the performance of a future action by another organism. As such, traces enable a flexible way to support indirect interaction between organisms without adopting dedicated communication channels'. Clinicians were then asked to share examples of traces they have used or have seen others use in their clinical context. Once specific examples were identified, participants shared additional insights around questions such as: How are traces used? What are the intended or unintended messages? What types of situations are they better suited for? Interviews were audio recorded, transcribed verbatim and deidentified by a professional transcription service to protect anonymity. In average, each interview produced 15-20 pages of data for analysis.

Data collection and analysis in CGT requires multiple readings of the dataset using an iterative processmeaning that data collection and analysis occurred simultaneously. ${ }^{32}$ This step involved both authors. The analytical process unfolded in three progressively interpretive stages as per CGT: initial, focused and theoretical. Initial coding-the first five transcripts were read line by line and coded using gerunds (action words ending in 'ing'), and participants' words (in vivo codes) to capture the meanings and actions described by participants. The intention of this first stage of analysis is to describe, rather than interpret, participants' perspectives and experiences to ensure that preliminary findings are firmly 'grounded' in the data. Focused coding-five more interviews were analysed and compared with the previous five to consolidate initial codes into preliminary themes that were used to focus code the rest of the transcripts. Theoretical coding - a coding framework (see online supplemental appendix 2) was finalised and used to recode the entire dataset. The results of this analytical process were presented to a subgroup of the participants to consider resonance, trustworthiness and transferability, ensuring the rigour of the analysis. ${ }^{35}$ Throughout this process, iteration and constant comparison, the two key principles of CGT, were central to the analytical approach. Since CGT does not start with an a priori theory, this study used traces as a sensitising concept when engaging in constantly comparing data within and between transcripts. Recruitment was ceased once theoretical sufficiency was achieved, ${ }^{36}$ meaning that the analysis determined that data categories offered sufficient conceptual depth and complex connections to fulfil the purpose of the study. Throughout the research process, reflective and analytical memos were written to move the findings from descriptive to interpretive to theoretical, and to document the author's engagement in reflexivity ${ }^{37}$ (see online supplemental appendix 3).

\section{Patient and public involvement}

Patients or the public were not involved in this study.

\section{RESULTS}

While participants did not label it as such, using traces for communication was not a foreign concept. Participants described trace-based communication and its elements in the healthcare setting in a variety of complex ways. We used these descriptions to develop a definition of a trace in a human social environment:

A trace is an object (mark, signal) that is intentionally and strategically used to modulate an environment in order to prompt action by another team member. Unlike non-human organisms, humans can interpret traces in different ways, such that the trace may succeed or fail to prompt the intended action, and may have multiple effects. A trace response is social, not biological, and is therefore influenced by time, space and interpersonal relations.

Using excerpts from study participants, the results will elaborate the following elements of the definition of a trace: intentionality, success/failure and response. In order to enhance readability, this section was written using the technique of integrating verbatim quotes from the participants into the description of the results. ${ }^{38}$ Italicised numbers in brackets represent the codes assigned to participants during the process of anonymisation. These numbers are also provided to comply with the authenticity principle of qualitative research writing. ${ }^{38}$

\section{Intentionality of a trace}

Regardless of the form that traces can take, participants took one of three positions when discussing the 
potential intentions behind them: promoting efficiency, preventing mistakes or saving face. In recalling an acute resuscitation, an emergency physician indicated that she felt the urge to position the ultrasound adjacent to the patient's chest to help the leader preserve flow by 'optimising the use of our [the team's] time' (110). Similarly, other participants talked about traces as a vehicle for efficiency, "when the nurse really wants us to hurry up and get a patient discharged, there will be an ophthalmoscope by the bedside' (113). In the neonatal intensive care unit, checking the eyes is usually something neonatologists do on discharge before a baby is to be transferred out. Conversely, others spoke about using traces to prevent lapses in patient care when they were interrupted by 'leaving all the tools at the bedside so that if the occupational therapist comes along, they will know that now would not be a good time to take them (the patient) away' (103). Yet other participants highlighted the potential of traces to be used 'as a face-saving thing, or a way of avoiding the patient seeing what I am thinking because I am not certain' (110). An example was the case of a paramedic who laid out two sets of equipment for his senior partner to indicate the correct equipment to use without having to verbalise it in front of the patient. This last intention was also recognised as a strategy to deal with 'issues of power and hierarchy to enhance or prevent a message to be communicated' (101). For instance, participants remarked that in teaching environments, 'they (traces) become socially more important when learners need to navigate ways to propose ideas and feel comfortable' (106) speaking their mind. This was particularly prevalent, for example, in settings where trainees rounded patients in multiprofessional teams with various levels of training, such as the clinical teaching units.

\section{Success and failure of a trace}

Traces were regarded as successful by participants when they triggered the intended action. For instance, a successful trace such as the 'butterfly, a little magnet or a sticker either on the chart or on the door' was commonly used in a neonatology unit. This trace prompted healthcare providers 'to be sensitive that there has been some loss experienced' (113). Two participants separately recalled feeling 'remarkably uncomfortable when hearing that pitch (the pulse oximeter) goes down (124); it is one of those traces that we reliably use because it is potent' (122) in indicating that a patient is losing oxygen rapidly and prompting the team to quickly reassess and take action. Participants also provided examples of failed traces when they realised the intended action was not achieved. Failed traces were identified as those that took on different meanings mainly due to a lack of attention as to how the evolution of the situation changed people's interpretations. For instance, local hospitals have created a new violence flagging policy where patients are given a purple arm band if they are considered a violence risk. However, with time, the purple arm band became a ubiquitous practice, prompting participants to describe it as a failed trace because 'we've actually diluted the signal by putting it (purple arm band) on everybody who's ever kind of been verbal or physical in a delirium' (116).

Other participants indicated that a trace also failed when they were ignored as when the intended message conflicted with personal preferences. For instance, in dealing with a colleague who tended to give more narcotics than what she felt necessary, one clinician described her approach of 'intentionally leaving a small syringe on the desk and leaving the rest tucked away in a corner' (117). Her goal was to convey the message that she wanted her colleague to only give so much. Despite her efforts, 'that cue was completely missed because (when) I came back the consultant said there was only so much in the syringe and he took the rest and gave the rest' (117). Another participant reflected that when things get busy, 'they (the consults) all get put back in the slot, so they had been laid out as a sign of, these need to get done, and the next thing I know they are back in the slot' (113) because people do not want to do the non-urgent consults and opt to undo the trace. Regardless of the potential for success or failure, participants also commented on the multiple effects that some traces carry. The most striking example was the purple armband that 'triggers a feeling of helplessness among staff who feel like our patients are being unfairly stigmatised', but who also believed that it should be 'part of workplace safety legislation' (112). Stigma and safety acted as two different effects of the purple arm band despite the original intention. When comparing the instances in which traces succeeded or failed, participants agreed in that it all came down to whether 'the individuals who are looking after that environment are used to understand the implications of it' (122) and able to respond accordingly.

\section{Situational responses to a trace}

Traces were described as being contextually bounded by time, space and interpersonal relationships. As participants described, particularly in highly acute situations, traces 'only really have a single meaning for the first 5-10 min' (122). If someone were to appear later, the chances for misinterpretation would increase. And this is because they missed the 'rest of the social interaction between the leader and the team members, (therefore) they are not going to be able to understand in what context it was put there'. As such, 'the power of the trace degrades because its meaning becomes unstable as time progresses and the social context progresses' (110). Another participant described it as the potential for losing the big picture, particularly when traces are left behind unintentionally or are moved to a different space. For instance, if after dealing with a traumatic cardiac arrest, the equipment remained in the resuscitation room and then a new cardiac arrest were to come in, it would be harder for the team members who maybe have not been present, who are new to the room, to gather a clear situational awareness of what is going on' (114). They might be left figuring out whether or not the resuscitation has 
already taken place likely losing precious time to deal with the acuity of the situation.

Time and space appeared as constraints for some participants. Yet for others, traces offered distinct benefits when comparing them to direct communication protocols. Some participants brought attention to the idea that when people refer to direct communication, they often refer to an interaction between two individuals. Whereas indirect communication using traces was described, in some instances, as being different, in that 'it is something that actually has a role within an environment' (125). As this participant illustrated, when someone uses a trace, they are not communicating to a single individual exclusively, "they are essentially inserting something into the environment... (to) include very contextualised pieces' that are not covered by a checklist because 'the checklist is generic' (125). An example in anaesthesia was the multiple ways in which an intubation is performed: "what (the tools) you use depends on what you are familiar with, but also on patient-related factors... there is nothing in the checklist that focus(es) your attention to what this patient needs'. In their opinion, 'it is not a failure of the checklist overall, it is a failure of the checklist to meet the needs of a complex situation' (125). This insight was used to ask subsequent participants to reflect on what other complex situations could be suitable for tracebased communication. They articulated four other: (1) emotionally consuming situations 'as in the labour and delivery ward, if there is a butterfly you don't go in and say congratulations, because you know there was a loss' (113); (2) low stakes situations that involve training where 'I may just kind of nudge the anaesthetic gel towards her... to kind of make them realise that they have forgotten it' (117); (3) situations where the core team is stable as in 'geriatric rehabilitation unit (where) it is much easier because everybody has worked with each other for some time' (118) and finally, (4) asynchronous situations such as when nurses prepare an operating room while the surgeon is in the wards and 'if nurses have concerns, they will leave the bedside chart in the room open' (119) to alert surgeons that something requires close attention. While a trace 'is probably most significant in the moment that it is used and then becomes less significant' (122), the same participant reflected on the idea that we should not discard traces outright because they have the capacity to flag the 'ways in which such environment changes' (122) as they are influenced by time, space and interpersonal relationships.

\section{An additional insight: hesitation while describing the use of traces}

Participants were hesitant and cautious when they described situations where they chose traces over verbal communication. Early in the interview process, the ambivalence that most participants experienced when recalling examples of traces was noticeable. When asked about it, they wondered if the reason why healthcare providers do not readily appreciate the benefits of traces is because 'our training is that you should never rely on indirect communication' (117). In their accounts, participants tended to add a cautionary note because 'I think we are really very much explicit communicators... we are almost engrained to not do anything that could be misinterpreted' (106). While this cautionary sense was not present in all interviews, the pervasive sense was that healthcare providers have been dissuaded from using non-verbal communication because of the assumption that 'indirect is most likely to get us into trouble' (103). This was an assumption that remained critically unquestioned by participants.

\section{DISCUSSION}

This study showed that while not fully endorsed, traces are pervasively used by healthcare professionals. Even though they are trained and prefer to use verbal communication, examples of traces abounded in this study. This disjuncture between preference and practice suggests that clinicians may have a blind spot for trace-based communication which might prompt them to discard it. However, discarding trace-based communication uncritically might be more dangerous than explicitly considering its pros and cons. Rather, acknowledging when it might be productive could contribute to the safety conversation in ways we have not considered. For instance, the operating room is an environment where non-verbal communication in the form of body language has found a productive place to balance urgency with patient care. ${ }^{3}$ Yet, non-verbal communication in the form of traces has not been identified or discussed in this environment. In fact, the few studies in healthcare that have hinted at tracebased communication have serendipitously arrived at it. In their exploration about barriers to speak up in anaesthesia, Beament and Mercer found participants using non-verbal challenges to complement high-grade verbal challenges. ${ }^{21}$ Within the repertoire of non-verbal challenges, trace-based communication to prevent further intubation took place in the form of removing the laryngoscope or placing the facemask in a particular place. The laryngoscope and the mask were used intentionally to gain someone's attention. While Beament and Mercer did not provide a particular discussion about the use of traces, they noted the need for further research. This study adds to this nascent conversation, demonstrating that trace-based communication constitutes another form of indirect communication used by healthcare providers. It further suggests that trace-based communication might prove beneficial for safety by improving healthcare teams' ability to collective adapt.

Given the intentionality ascribed to traces, traces carry symbolic meaning. ${ }^{13}$ The practical use of traces within a given social context (eg, operating rooms, intensive care units, clinical wards, etc) create meanings that can be shared by others. This is referred to as intersubjectivity. ${ }^{39}$ Some of the participants described traces, such as the butterfly, that had become somewhat standardised within their contexts. This standardisation seemed particularly 
important for coordinating activities. As elaborated by participants, the butterfly trace created a symbolic repertoire ${ }^{13}$ in the form of a behavioural routine that all members of the team abided by. While a key advantage of creating this kind of symbolic repertoire is to support explicit communication, team members need to remain attuned to counteracting its potential risks. For instance, when new team members join, they should be explicitly trained to use the symbolic repertoire in order to prevent unintentional mistakes when the team needs to rely on non-verbal communication. Training for identification of traces and their assigned meaning might constitute an important effort in improving a team's ability to effectively communicate.

By studying trace-based communication in a human social context such as healthcare teamwork, this study also elaborated the construct of a trace. Focused intentionality, successful versus failed traces and the contextually bounded nature of the responses to traces (time, space and interpersonal relationships) were brought to light in this novel conceptualisation. The literature on trace-based communication in non-healthcare contexts has focused on its applications. Little conceptual work has been done to deconstruct the unique features of traces as they are used in human contexts. Tummolini and Castelfranchi's ${ }^{40}$ work emerged as likely the first and only attempt thus far. Their main contribution is a taxonomy of basic messages that can be communicated when using traces in daily social life. Those basic messages were classified around seven purposes: informing about presence, intention, opportunity for action, action accomplished, goal or result. The authors further suggest that while basic, these messages can be combined into complex behavioural messages, such as using a trace to deliver multiple messages. Although offered as a conceptual framework without empirical evidence, their work contains useful language about the general uses of a trace by humans. ${ }^{40}$ To add to this body of literature, the present study uncovered two key intentions in the use of traces in the context of healthcare: promoting efficiency and preventing mistakes. Tummolini and Castelfranchi also asserted that given our human nature, different meanings can coexist in the same trace. To advance this notion, participants in the present study elaborated further to illustrate how traces succeed or fail depending on whether or not the triggered response takes place within certain temporal, spatial and social conditions. In the absence of extensive conceptual work and the recent calls for more nuanced characterisations, ${ }^{23}$ the empirical evidence provided by the present study speaks to the theoretical conversations of what traces are and how they are being used and responded to in relation to the context.

From a practical perspective, participants in this study acknowledged using trace-based communication, yet they expressed hesitance and provided several cautionary tales. Other industries have moved past the hesitancy to embrace the usefulness of trace-based communication. The military offers the best example. ${ }^{41}$ As in the present study, the military has discovered that trace-based communication is useful in some situations and not others. For instance, in ambush situations where safety is paramount because of the asynchronous nature of work, groups of Navy SEALs are currently trained to be attuned to how other team members' actions modify the environment. In a similar way, in this study participants found trace-based communication particularly useful in high-stakes asynchronous situations; they also identified a range of other situations where trace-based communication might be useful, including training situations, emotionally heightened situations and situations where team instability was not a threat. As one might anticipate, not all situations are amenable for trace-based communication. In the military, trace-based communication is avoided in briefing/ debriefing situations. ${ }^{414}$ The same should be expected in healthcare, for instance in contexts such as trauma, where post-mortem conversations are deemed essential for teamwork.

Whether pervasive or not, this study showed that traces might play a key role in the ability of teams to maintain situational awareness. As illustrated by participants' examples, traces afford the ability to anticipate the leader's and team's needs without disrupting flow. Therefore, a practical recommendation from this study is that healthcare providers engage in simulation training and debriefing conversations that explicitly examine moments in which team members have used a trace to convey a message. By bringing trace-based communication to the surface of those conversations, it is possible to clarify intentionality and uncover patterns of behaviour towards improving team situational awareness. Furthermore, as others have suggested, simulation training offers the opportunity to combine both verbal and non-verbal communication, including trace-based communication, in patient safety scenarios. $^{7}$

\section{LIMITATIONS}

As with any empirical study, the findings presented here are bound by research design decisions that draw attention to certain aspects and deflect attention from others. For instance, the decision to sample clinicians from a variety of specialties offered the opportunity to identify patterns in how traces are used in clinical environments but prevented exploring in detail which environments may benefit more than others. Future work could explore the organisational and cultural characteristics of particular clinical environments that influence a trace's success or failure.

While this paper treated non-verbal communication as a separate entity to bring awareness to forms of non-verbal communication other than body language, it is important to note that that verbal and non-verbal communication go hand in hand as communication theory has argued. ${ }^{11} 12$ Therefore, further research is needed to investigate how trace-based communication is used in combination with verbal communication and their influences on how teams work. A context where this exploration might be highly 
relevant is interprofessional teamwork communication (eg, communication between surgery and anaesthesia teams). Using the definition offered in this study, methods such as field observations and simulation, in addition to interviews, would add valuable insights into how multiple teams use and respond to traces.

Finally, this paper focused on providing a general definition of a trace and consequently, other aspects were not elaborated, such as how traces are created or categorised. For instance, some participants suggested certain traces as being institutionally sanctioned and others as being created informally out of their personal intentions. While such distinction was outside the focus of this paper, it will be important to consider in future research in order to better understand how and why the way traces are created modulate and influence communication dynamics among team members and across teams.

\section{CONCLUSION}

This study has demonstrated that trace-based communication is pervasive in clinical work, but that its conceptualisation had been largely ignored. As such, this study was also intended to plant a seed to at least begin the conversation around trace-based communication. Because unless we take the time to explore it more systematically, we will not be able to decide whether it has any place in our efforts to enhance teamwork and communication for safety.

\section{Twitter Sayra Cristancho @scristaM}

Contributors SC designed the study, collected the data and led the writing of the manuscript. Both SC and EF conducted data analysis and EF further participated in writing for the revised manuscript.

Funding This work was supported by Physicians Services Incorporated Foundation in the grant category 'Health Research Grants'. Grant title: Building the collective competence of an interprofessional team.

Competing interests None declared.

Patient consent for publication Not required.

Provenance and peer review Not commissioned; externally peer reviewed.

Data availability statement Data are available upon reasonable request. Part of the anonymised data is shared in Appendix 2 to illustrate the coding framework used for data analysis.

Supplemental material This content has been supplied by the author(s). It has not been vetted by BMJ Publishing Group Limited (BMJ) and may not have been peer-reviewed. Any opinions or recommendations discussed are solely those of the author(s) and are not endorsed by BMJ. BMJ disclaims all liability and responsibility arising from any reliance placed on the content. Where the content includes any translated material, BMJ does not warrant the accuracy and reliability of the translations (including but not limited to local regulations, clinical guidelines, terminology, drug names and drug dosages), and is not responsible for any error and/or omissions arising from translation and adaptation or otherwise.

Open access This is an open access article distributed in accordance with the Creative Commons Attribution Non Commercial (CC BY-NC 4.0) license, which permits others to distribute, remix, adapt, build upon this work non-commercially, and license their derivative works on different terms, provided the original work is properly cited, appropriate credit is given, any changes made indicated, and the use is non-commercial. See: http://creativecommons.org/licenses/by-nc/4.0/.

\section{ORCID iD}

Sayra Cristancho http://orcid.org/0000-0002-8738-2130
REFERENCES

1 Etherington N, Wu M, Cheng-Boivin O, et al. Interprofessional communication in the operating room: a narrative review to advance research and practice. Can J Anaesth 2019;66:1251-60.

2 Mitchell L, Flin R, Yule S, et al. Thinking ahead of the surgeon. An interview study to identify scrub nurses' non-technical skills. Int $J$ Nurs Stud 2011;48:818-28.

3 Moore A, Butt D, Ellis-Clarke J, et al. Linguistic analysis of verbal and non-verbal communication in the operating room. ANZ J Surg 2010;80:925-9.

4 Schwappach DLB, Gehring K. 'Saying it without words': a qualitative study of oncology staff's experiences with speaking up about safety concerns. BMJ Open $2014 ; ; 4:$ :e004740

5 Hill K. The sound of silence - nurses' non-verbal interaction within the ward round. Nurs Crit Care 2003;8:231-9.

6 Manojlovich M. Nurse/physician communication through a sensemaking lens: shifting the paradigm to improve patient safety. Med Care 2010;48:941.

7 Härgestam M, Hultin M, Brulin C, et al. Trauma team leaders' non-verbal communication: video registration during trauma team training. Scand J Trauma Resusc Emerg Med 2016;24.

8 Schöpf AC, Martin GS, Keating MA. Humor as a communication strategy in Provider-Patient communication in a chronic care setting. Qual Health Res 2017;27:374-90.

9 Bergman AA, Jaynes HA, Gonzalvo JD, et al. Pharmaceutical role expansion and developments in Pharmacist-Physician communication. Health Commun 2016;31:161-70.

10 Gordon DM. Ants at work: how an insect Society is organized. Simon and Schuster, 1999: 202.

11 Jones SE, LeBaron CD. Research on the relationship between verbal and nonverbal communication: emerging integrations. J Commun 2002;52:499-521.

12 Kendon A. Studies in the behaviour of social interaction. Bloomington: Indiana University Press, 1977.

13 Duncker E. Symbolic communication in multidisciplinary cooperations. Sci Technol Human Values 2001;26:349-86.

14 Litak D. Colour coding scrubs as a means of improving perioperative communication. J Perioper Pract 2011;21:177-8.

15 Silverman J, Kinnersley P. Doctors'non-verbal behaviour in consultations: look at the patient before you look at the computer. $\mathrm{Br}$ J Gen Pract $2010 ; ; 60: 76-8$

16 Marcinowicz L, Konstantynowicz J, Godlewski C. Patients' perceptions of GP non-verbal communication: a qualitative study. $\mathrm{Br}$ $J$ Gen Pract 2010;60:83-7.

17 Hall JA, Harrigan JA, Rosenthal R. Nonverbal behavior in clinicianpatient interaction. Appl Preven Psychol 1995;4:21-37.

18 Roter DL, Frankel RM, Hall JA, et al. The expression of emotion through nonverbal behavior in medical visits. J Gen Intern Med 2006;21:28-34.

19 Collins LG, Schrimmer A, Diamond J, et al. Evaluating verbal and non-verbal communication skills, in an ethnogeriatric OSCE. Patient Educ Couns 2011;83:158-62.

20 Graham SL. Hospitalk: politeness and hierarchical structures in interdisciplinary discharge rounds. J Politen Res 2009;5:11-31

21 Beament T, Mercer SJ. Speak up! barriers to challenging erroneous decisions of seniors in anaesthesia. Anaesthesia 2016;71:1332-40.

22 Burgoon JK, Guerrero LK, Manusov V. Nonverbal communication. Routledge, 2016: 595.

23 Susi T, cognition S. Social cognition, artefacts, and stigmergy revisited: concepts of coordination. Cogn Syst Res 2016;38:41-9.

24 Garnier S, Gautrais J, Theraulaz G. The biological principles of Swarm intelligence. Swarm Intell 2007;1:3-31.

25 Woolley-Barker T. Teeming: how superorganisms work together to build infinite wealth on a finite planet (and your company can too). White Cloud Press, 2017: 224.

26 Arquilla J, Ronfeldt D. Swarming and the future of conflict [online]. Santa Monica: RAND Corp, 2000. https://apps.dtic.mil/docs/ citations/ADA384989

27 Christensen LR. Stigmergy in human practice: coordination in construction work. Cogn Syst Res 2013;21:40-51.

28 Rosenberg L, Lungren M, Halabi S, et al. Artificial swarm intelligence employed to amplify diagnostic accuracy in radiology. In: 2018 IEEE 9th annual information technology, electronics and mobile communication conference (IEMCON), 2018: 1186-91.

29 Butler JI, Fox MT. Nurses' perspectives on interprofessional communication in the prevention of functional decline in hospitalized older people. Health Commun 2019;34:1053-9.

30 Pope C, van Royen P, Baker R. Qualitative methods in research on healthcare quality. Qual Saf Health Care 2002;11:148-52.

31 Cristancho SM, Goldszmidt M, Lingard L, et al. Qualitative research essentials for medical education. Singapore Med J 2018;59:622-7. 
32 Charmaz K. Constructing grounded theory. Sage, 2014.

33 Coyne IT. Sampling in qualitative research. purposeful and theoretical sampling; merging or clear boundaries? J Adv Nurs 1997;26:623-30.

34 Ritchie J, Lewis J, Nicholls CM, et al. Qualitative research practice: a guide for social science students and researchers. SAGE, 2013: 457.

35 Birt L, Scott S, Cavers D, et al. Member checking: a tool to enhance Trustworthiness or merely a NOD to validation? Qual Health Res 2016;26:1802-11.

36 Nelson LH. The very idea of feminist epistemology. Hypatia 1995;10:31-49.

37 Finlay L. "Outing" the researcher: the provenance, process, and practice of reflexivity. Qual Health Res 2002;12:531-45
38 Lingard L. Beyond the default colon: effective use of quotes in qualitative research. Perspect Med Educ 2019;8:360-4.

39 Husserl DA. intersubjectivity and anthropology. Anthropological Theory 2010;10:16-35.

40 Tummolini L, Castelfranchi C. Trace signals: the meanings of stigmergy. In: Weyns D, Parunak HVD, Michel F, eds. Environments for multi-agent systems III. Berlin Heidelberg: Springer, 2007: 141-56.

41 Zweibelson B. Let me tell you about the birds and the bees: Swarm theory and military decision making. Canadian Military J;15:29-36.

42 Coyle D. The culture code: the secrets of highly successful groups. Random House Publishing Group, 2018: 305. 